\title{
INNOVACIONES EDUCATIVAS: PRÁCTICAS ORIENTALES APLICADAS A LA EDUCACIÓN A TRAVÉS DEL ARTE.
}

Silvia Viñao1: Universidad de Granada. España

silviavinao@gmail.com

\section{RESUMEN}

Determinadas técnicas orientales se utilizan cada vez con más frecuencia e de forma experimental en procesos de intervención educativa. Tai chi, meditación, yoga,..., pueden ser excelentes recursos que educadores, maestros y animadores emplean en proyectos educativos. Este artículo versa sobre esta cuestión aplicándola a la educación a través del arte, con el fin de explorar las potencialidades que encierran este tipo de prácticas y procesos.

PALABRAS CLAVE: Arte - Educación - Innovación educativa - Meditación - Tai chi.

\section{EDUCATIONAL INNOVATIONS: ORIENTAL PRACTICES APPLIED TO EDUCATION THROUGH ART}

\begin{abstract}
Some Oriental techniques are increasingly used, in a experimental way, in education processes. Tai chi, meditation, yoga, ..., all of them have become excellent means that educators, teachers and socio-cultural coordinators, use in their educational projects. This text deals with this issue applying it to education through art, in order to explore to the possibilities offered by this kind of practices and processes.
\end{abstract}

KEY WORDS: Art - Education - Educational Innovation - Meditation - Tai chi

\footnotetext{
${ }^{1}$ Autor Correspondiente

Silvia Viñao: Doctora por la Universidad de Granada. Licenciada en Bellas Artes

Correo: silviavinao@gmail.com
} 


\section{INTRODUCCIÓN}

\section{Contextualización}

Es interesante comprobar cómo las innovaciones educativas están enriqueciéndose actualmente con aportaciones procedentes de otras culturas. Esta es una consecuencia lógica para quien realmente tiene un espíritu investigador alejado del sentimiento colonialista, según el cual nuestra cultura se percibe como "la cultura", es decir la cultura universal, más aún cuando existen culturas milenarias como la china.

Dicha cultura alcanzó un gran desarrollo en campos como la filosofía o el arte, y concedió desde sus comienzos una gran importancia a la necesidad de educar. En mi tesis doctoral Del arte a la educación: aportaciones plásticas y pedagógicas de la estética taoísta he tenido el placer de investigar la cultura china, e incluso de realizar algunas experiencias empíricas basadas en las técnicas de la meditación y el tai chi.

En esta disertación abordaremos dichas experiencias en las cuales comprobaremos la utilidad, o no, de la meditación y el tai chi, que pueden servir como medio para enriquecer la educación a través del arte. Como en este campo lo importante no es el dibujo sino el dibujante, no nos perderemos en exponer las técnicas de pintura china, sino que nos centraremos en el proceso de modificación de la percepción de observador o del dibujante.

Comenzaremos con un breve repaso a las nuevas corrientes pedagógicas en las cuales científicos, pedagogos y psicólogos, investigan sobre el impacto de la técnica de la meditación en el ser humano y sus posibles aplicaciones pedagógicas. Seguidamente expondremos un resumen de las pruebas empíricas realizados durante la realización de mi tesis doctoral. La primera, indaga sobre los efectos de la meditación en la contemplación de la obra de arte y la segunda en las aportaciones del tai chi a la pincelada y al artista. Por último, se expondrán las conclusiones extraídas. La metodología empleada en estos ejercicios es fundamentalmente cualitativa, empleándose también el método de las notas de campo y la observación participante.

\section{METODOLOGÍA}

La base metodológica se basa en el análisis de las anteriores escuelas de pensamiento que han tratado el tema que nos ocupa. Se trata de analizar los principales medios existentes hoy y aplicarles un método analítico textual que permita producir inferencias fenomenológicas o estructuras de pensamiento novedoso basado en este tipo de reflexiones que crean un puente entre las ideas anteriores (y sus realidades descritas) y las más modernas. 


\section{ANÁLISIS Y DISCUSIÓN}

\subsection{Nuevas corrientes pedagógicas}

Desde una mentalidad ortodoxa de la enseñanza este experimento puede parecer arriesgado, o cuanto menos sin sentido, pero tenemos que ampliar nuestro concepto de la enseñanza y de su metodología si queremos conseguir una reforma o evolución profunda de ambas, sobre todo si pretendemos influir positiva y sustancialmente en la formación de nuestros alumnos como individuos y como seres humanos.

Afortunadamente pedagogos y científicos ya han realizado experiencias prácticas similares empleando técnicas de meditación en las aulas, como podemos comprobar en el programa Redes, número 50, titulado "Meditación y aprendizaje". En dicho programa se destaca la necesidad de crear un nuevo sistema educativo que se destine no solo a formar trabajadores sino también ciudadanos.

Con este fin pedagogos y científicos se reunieron en Washington con el Dalai Lama para debatir sobre la incidencia de la meditación en el ser humano y su aplicación en la educación. Según Punset (Punset,2009):

Los científicos han descubierto que por primera vez el impacto de la meditación, de las técnicas meditativas, sobre el estado, sobre la esencia, sobre la conciencia de un niño de un joven o de un adulto, no sabemos todavía en que medida le afecta, pero sí conocemos ya que le afecta.

Como ejemplo citaremos el experimento empírico del psicólogo Mark Greenberg, que realizó un estudio preliminar sobre "yoga y presencia alerta" en las escuelas de Baltimore con niños de 10 y 11 años. Los niños realizaban entre 30 y 40 minutos de yoga y meditación, y como resultado Greenberg constató que el yoga y la meditación redujeron lo que él denomina el "rumiar" de la mente, los pensamientos intrusivos, y mejoró la habilidad de los niños para manejar sus emociones.

Además, en dicho programa se destaca cómo la meditación enseña a focalizar la atención y ayuda al autocontrol y autoconocimiento. En síntesis, el programa corrobora muchos de los argumentos defendidos en esta investigación. Siguiendo esta línea, realizaremos algunos ejercicios que servirán de muestra sobre cómo la meditación, y los ejercicios de origen taoísta o chan, pueden aplicarse a la educación a través del arte. 


\subsection{Pruebas empíricas realizadas}

Las posibilidades que se nos presentan a este respecto son tan variadas como ilimitadas, ya que los tipos de meditación o ejercicios meditativos son múltiples y las edades a las que aplicarlos también. En este sentido, hemos escogido dos tipos de ejercicios: uno contemplativo y otro activo, ya que de este modo abarcamos dos facetas fundamentales del arte: la apreciación de una obra de arte y la creación artística.

\subsection{Primer ejercicio: Contemplación para la apreciación de la obra de arte}

El primer ejercicio es una prueba destinada a mejorar la apreciación de la obra de arte. Para abreviar, nos referiremos al mismo como ejercicio de "contemplación". Su objetivo es constatar cómo la meditación influye en el estado de ánimo de la persona y cómo el estado de ánimo en el que ésta se encuentra repercute a su vez en la forma de observar o contemplar la obra de arte.

En una sociedad en la que llevamos un ritmo de vida tan acelerado, cuando vamos a una sala de arte o a un museo no le concedemos a la obra de arte el tiempo necesario para su contemplación, ni en ocasiones la actitud adecuada. En la estética taoísta la contemplación es un arte, una degustación de la obra, que requiere su tiempo y un estado de ánimo adecuado. Por ello es interesante realizar una experiencia empírica en la cual se puedan comprobar los efectos de la meditación en la percepción de la obra de arte. El ejercicio de "contemplación" se llevó a cabo con jóvenes universitarios de unos de 18 a 33 años de edad aproximadamente. La mayoría nunca habían realizado prácticas de meditación, y ni un ejercicio de este tipo.

Este margen de edad parecía ser el idóneo para este ejercicio, ya que con alumnos más jóvenes realizar un ejercicio de meditación por primera vez, sin dar unas clases previas de meditación o relajación, podía ser más complicado.

\subsection{Desarrollo del ejercicio}

En primer lugar, un grupo de 10 alumnos entraba en el aula donde se habían colocado, en unas sillas en círculo, 10 acuarelas originales que representaban diferentes paisajes.A los alumnos les dije que imaginasen que entraban en una sala de exposiciones y que dieran vueltas en círculo mirando las acuarelas. Cada vez las vueltas debían ser más rápidas, e incluso les hacia cambiar el sentido de la marcha; esto lo hice para que la elección de la obra fuese casual.

Cuando les decía "stop", cada alumno se quedaba quieto delante de la acuarela que le hubiera tocado en suerte, la observaban unos minutos, y de pie frente a la acuarela escribían lo que ésta les transmitía durante unos 5 o 10 minutos, según necesitaran. Luego debían darle la vuelta a la acuarela de manera que ya no la vieran. 
En la segunda fase de este ejercicio los alumnos debían realizar una técnica de meditación sencilla: sentados en un cojín con una postura de meditación cómoda (es decir, con la piernas cruzadas y la espalda recta) debían concentrarse en su respiración. Para ello podían contar cada vez que el aire entrase o saliera de sus fosas nasales, para luego, una vez más concentrados, observar este proceso dejando de contar cuando ellos lo creyeran conveniente.

Primero hicimos una prueba de 3 o 4 minutos, después de la cual podían decir si habían tenido algún problema, pues había unos pocos alumnos a los que les costaba relajarse, se mareaban o les daba risa o incluso picores. Después de darles algunos consejos para relajar la mente hicimos una meditación de unos 10 minutos aproximadamente. Mientras estaban sentados con los ojos cerrados, colocaba la acuarela de tal modo que al abrir los ojos fuese lo primero que vieran frente a ellos. Cuando les dije que abrieran los ojos, les indiqué que siguieran observando en el mismo estado de relajación. Se quedaban así unos minutos y luego comenzaban a escribir lo que les sugería la acuarela o seguían observando, si querían, durante 5 o 10 minutos, según necesitasen.

Naturalmente, para la correcta realización del ejercicio la acuarela debía ser la misma en la primera observación y en la segunda. Las 10 acuarelas empleadas representaban paisajes de un tamaño de $50 \times 35 \mathrm{~cm}$. aproximadamente, realizadas por mí aunque no expresamente para este ejercicio. Escogí estas acuarelas, en primer lugar, porque me pareció importante que fuese obra original y poseer originales de otros artistas no es fácil, y, en segundo lugar, porque mi estilo no es ni realista ni abstracto y quería un estilo pictórico que sugiriera, es decir donde las formas no fuesen del todo realistas, con el fin de poder extraer una mayor variedad de lecturas. Como ejemplo mostraré uno de los casos recogidos a partir de las experiencias con las diez acuarelas, en concreto con la número 5.

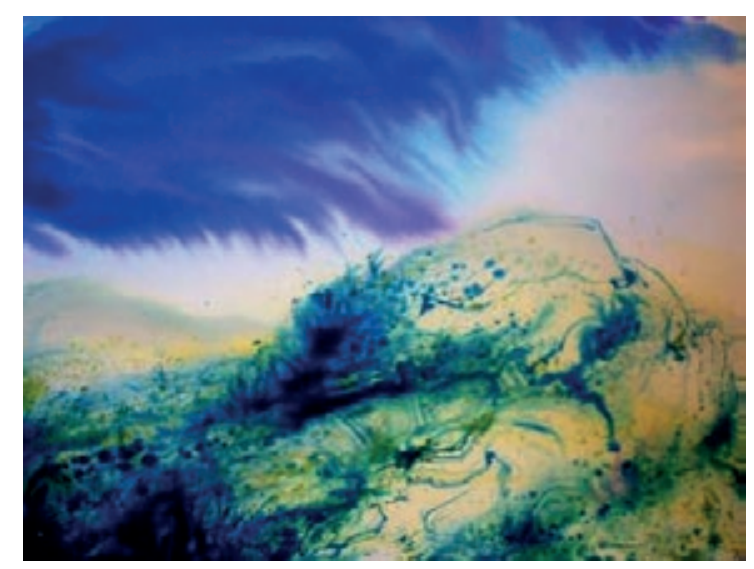

Figura 1. Acuarela número 5. 


\subsection{Resultados del ejercicio}

Este ejercicio se realizó tres veces con tres grupos de 10 alumnos cada uno. En general, en todos los grupos el tipo de percepciones de la obra observada, antes y después de la meditación varió, en la mayoría de los casos, ya sea en mayor o menor medida.

Las explicaciones sobre lo que les había transmitido la obra antes de la meditación eran en general más descriptivas, y cuando hacían referencia a las sensaciones o los sentimientos que la obra les transmitía eran en general de soledad, inquietud, frío, viento, agobio, tristeza, furia en el cielo, y en algunos pocos casos de relajación o esperanza. Excepcionalmente también aparecía alguna sensación positiva.

Después de la meditación disminuían notablemente los sentimientos negativos, apareciendo rara vez los de agobio o inquietud. Seguía persistiendo el sentimiento de soledad, pero esta vez sin un matiz negativo (una soledad en la que uno se encuentra bien), y sobre todo aumentaron considerablemente las percepciones de tranquilidad, calma, paz, plenitud e incluso pureza. También se incrementaron sensaciones positivas tales como la esperanza o la satisfacción. Asimismo, varió su percepción plástica: en general, admitían que después de la meditación veían o se fijaban en más detalles. Algunos, nada más abrir los ojos, se quedaban mirando una zona del cuadro, y otros decían que los colores les parecían más vivos.

Generalmente los escritos realizados después de la meditación, tenían un carácter más profundo, contenían más referencias emocionales y eran menos descriptivos. Podríamos decir por tanto, que la resonancia de la obra en el espectador aumentaba. Es curiosa la observación de algunos pocos alumnos que expresaban la sensación, en la primera observación, de que parecía que iba a llegar una tormenta y, en la segunda, de que la tormenta ya había pasado, que se abría el cielo y de que esto les producía una sensación de esperanza.

Como ejemplo mostrare el caso de una alumna, Isabel Illán Herráiz, que observó la acuarela número 5. Comenzaremos observando las palabras escritas por la alumna antes de la meditación: El dibujo me transmite lo oscuro, la muerte, una vegetación sin vida a causa de la escasez del sol y agua. Me parece muy interesante y que transmite mucho.

La misma alumna frente a la misma acuarela, después de la meditación, escribió:

Observo que tras la muerte de la vegetación aparecen flores, con vida. También parece que la nube que tapa el sol se está haciendo a un lado para que los rayos hagan brotar vida. Me transmite que después de una muerte siempre hay más vida. Creo que es un dibujo muy interesante, que transmite mucho. 
Hay que reconocer también que no todas las sensaciones descritas por los alumnos son tan esclarecedoras. No obstante, como observación adicional podemos añadir que los alumnos salieron satisfechos del ejercicio; algunos manifestaban haber sentido que algo parecido ocurría en la vida. Según sus propias palabras, cuando uno está en positivo ve las cosas mejor, añadiendo que habían aprendido una lección.

\section{CONCLUSIONES}

Basándonos en los resultados obtenidos en el análisis total de los resultados de los tres grupos, podemos extraer que después del ejercicio de meditación se incrementan la profundidad de la percepción y las sensaciones positivas, sobre todo las de paz y serenidad. Estos estados de ánimo son aquellos en los que uno se encuentra después de meditar. Las manchas y los colores en el cuadro son los mismos; sin embargo después de la meditación, aunque en el cuadro se representen nubes de tormenta, éstas ya no se ven como una amenaza. Por tanto, también podemos concluir afirmando que el espectador percibe según su estado de ánimo, y que a su vez proyecta su estado de ánimo en lo percibido; es decir, que se produce una empatía entre el observador y la obra contemplada. Empatía o resonancia que, como hemos podido comprobar, es más profunda a nivel emocional después del ejercicio de meditación. Por tanto, podemos afirmar que la meditación si influye en la apreciación de la obra de arte.

No es de extrañar, por tanto, que los taoístas o budistas chan realizasen ejercicios meditativos, y que además demostrasen una especial sensibilidad hacia el arte o la naturaleza, ni que una de las claves de la estética taoísta sea la resonancia o empatía. Con este ejercicio se demuestra lo mucho que queda por aprender y descubrir de sus métodos, tal y como se apreciará asimismo en el próximo ejercicio basado en otra práctica milenaria china: el tai chi.

\subsection{Segundo ejercicio: Relación entre el tai chi y la pincelada}

Siguiendo esta línea, el segundo ejercicio, al cual denominaremos "Relación entre el tai chi y la pincelada", tenía como objetivo descubrir hasta qué punto los movimientos del tai chi, y la concentración en el propio cuerpo que esta disciplina exige, pueden influir en la pincelada del artista.

La elección del arte del tai chi no es casual. Con el tai chi se trabaja la autoconciencia del movimiento de la respiración y del equilibrio, concentrando la atención en el propio cuerpo (Al Huang, 2002). No obstante, cuando pintamos, al menos en Occidente, nuestra atención se dirige casi exclusivamente al resultado visual de la obra. Normalmente las pinceladas las damos de acuerdo con un patrón visual preconcebido, ya sea un boceto, la copia de un modelo o sólo una idea mental, que también es visual. 
¿Qué ocurriría si trazáramos las pinceladas sin un patrón visual previo, centrándonos sólo en los movimientos del tai chi? ¿Conseguirían los movimientos de tai chi dar como resultado una pincelada fluida y sin titubeos?

En términos de Gardner (Gardner, 1994), ahora vamos a trabajar con la inteligencia corporal, que es lo que hacemos con el tai chi. Con el tai chi tomamos conciencia del movimiento de nuestro cuerpo y de su fluidez o falta de ella, pudiendo así corregirlo, al tiempo que físicamente conseguimos una mayor elasticidad, agilidad, equilibrio y coordinación. Este tipo de cualidades son tremendamente útiles para cualquier persona, pero para un artista que valore la fluidez y coordinación de sus movimientos a la hora de pintar, creemos que todavía es más importante como a continuación intentaremos demostrar.

Cada vez que ejecutamos un movimiento de tai chi nuestros brazos describen un movimiento generalmente circular o al menos curvo. Si llevásemos un pincel en las manos mientras hacemos tai chi, y éstas dejasen el rastro del pincel en una superficie, el pincel describiría un trazo, una pincelada, que plasmaría la orbita trazada por la mano.

Desde mis comienzos de la práctica del tai chi, esta idea (como no es de extrañar en una pintora) me rondó la cabeza. En un primer momento, pensé en realizar este ejercicio basándolo sólo en mi experiencia, pero advertí que al analizar mis trazos seria difícil delimitar donde empieza la influencia del tai chi y donde la de años de práctica pictórica. Por ello, decidí dar un paso más: ¿qué ocurriría si un experto en tai chi, sin formación ni afición artística, cogiera unas brochas y, siguiendo los movimientos del tai chi, trazara unas pinceladas?

Plantear el ejercicio práctico a partir de esta pregunta me parecía más correcto, ya que si queremos demostrar que los movimientos del tai chi por si solos, como fruto de una disciplina o de un arte oriental, dan como resultado una pincelada fluida y precisa, sin titubeos ni cortes, es mejor demostrarlo con alguien que se dedica a esta disciplina y carece de formación artística. La persona escogida fue Pedro Sanlázaro, profesor de tai chi durante más de treinta años.

\subsection{Desarrollo del ejercicio}

Durante la primera fase del ejercicio, Pedro y yo estuvimos seleccionando los movimientos de tai chi idóneos para esta prueba empírica, ya que no todos eran fácilmente adaptables. La principal dificultad con la que nos encontramos es que el tai chi se realiza en un espacio tridimensional, por tanto los movimientos son tridimensionales, pero ahora teníamos que adaptarlos para que dejasen un rastro en una superficie plana. 


\subsection{Ejercicio de tai chi $\mathrm{N}^{\circ} 1$ : "La cigüeña blanca extiende sus alas"}

Teniendo esta dificultad en cuenta, elegimos la secuencia de movimientos denominada "La cigüeña blanca extiende sus alas". Los materiales empleados durante todos los ejercicios fueron brochas, tinta china diluida y papeles de estraza. En este ejercicio Pedro empleó sólo una brocha que manejó con la mano derecha. Seguidamente mostramos el resultado del ejercicio en la figura 1.

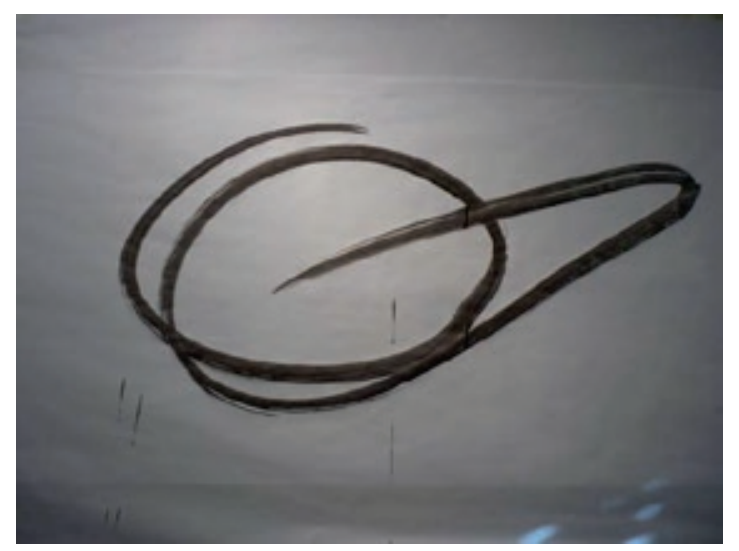

Figura 2. Resultado de la secuencia de movimientos denominada

“La cigüeña blanca extiende sus alas".

Como podemos observar en el resultado, el trazo es seguro y sin titubeos, y las elipsis están definidas con una pincelada resuelta y fluida. Aunque el trazo no represente ninguna imagen real, si analizamos su fluidez y la decisión con la que ha sido trazado, vemos que son las propias de un artista con bastante soltura en el manejo del pincel. En este sentido tengo que recalcar que Pedro no sabía ni cargar correctamente el pincel y que tuve que indicarle como hacerlo. Es decir, carecía totalmente de conocimientos y de práctica pictórica.

Con el fin de entender mejor la realización de este ejercicio, añadiré que, justo antes de llevarlo a cabo, Pedro me estuvo comentando que estaba concentrado en su Tan Tien y en los movimientos de su pelvis, y que el resultado visual no le importaba.

\subsection{Ejercicio de tai chi $\mathrm{N}^{0}$ 2: "Final de la cigüeña"}

Después de haber realizado dos ejercicios en los que predominaban las líneas curvas, decidimos realizar uno que diera como resultado líneas rectas.Por esta razón, el siguiente movimiento escogido fue el final de la secuencia de movimientos denominada "la cigüeña blanca extiende sus alas". 
Este ejercicio fue realizado tomando primero el pincel con la mano derecha y, una vez ejecutado el movimiento con esta mano, hacerlo a la inversa con la mano izquierda, repitiendo el mismo movimiento en sentido contrario. Con el fin de obtener una mayor concentración en el movimiento corporal, el ejercicio fue realizado por Pedro con los ojos cerrados.

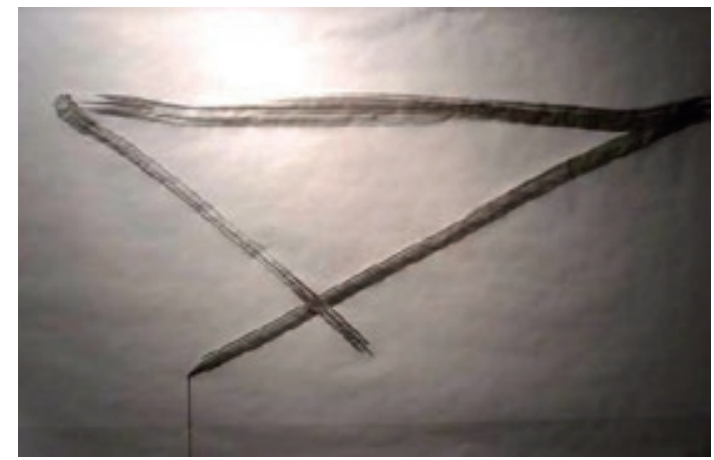

Figura 3. Resultado de la fase final de la cigüeña, realizada dos veces en sentido inverso.

En la Figura 3 comprobamos como, al tratarse de una figura más geométrica, podemos observar cómo se consigue una cierta simetría, cómo la inclinación de las verticales coincide en ambos trazos, y cómo los trazos horizontales se unen en una sola línea. Si tenemos en cuenta que el ejercicio ha sido realizado con los ojos cerrados, queda demostrado que la concentración en los propios movimientos del cuerpo da como resultado una pincelada fluida y segura.

En los días posteriores seguimos realizando diversos ejercicios con resultados similares. La mayoría de los ejercicios fueron realizados por Pedro, finalmente yo también me decidí a realizar algunos, pudiendo comprobar muchas de las cuestiones anteriormente descritas.

Por último, podemos destacar que la práctica de los movimientos del tai chi sí influye en la pincelada confiriéndole una mayor fluidez y decisión. Estas características se transmiten hasta tal punto que incluso los trazos realizados con la mano izquierda en personas diestras tienen la misma fluidez que los trazos realizados con la mano derecha.

Así pues, con esta prueba empírica quedan demostradas las ventajas de la práctica del tai chi aplicadas a la pintura, y concretamente su influencia sobre la pincelada. Además no viene mal recordar que estas ventajas no sólo se transfieren a la pincelada, sino al propio artista o practicante de tai chi que gana en movilidad, agilidad, equilibrio, concentración y autoconciencia, porque, como llevamos manteniendo hasta ahora, lo importante no es el dibujo sino el dibujante. Lo que ocurre es que cuando cambia el dibujante cambia el dibujo, y la forma más evidente de 
Finalmente, esperamos que estos experimentos empíricos sirvan para acercarnos un poco más a las disciplinas orientales que pueden enriquecer tanto la práctica artística del artista, ya sea profesional o aficionado, como la práctica educativa. Las técnicas del tai chi y la meditación llevan practicándose durante siglos en China y en otros países orientales, y han llegado a Occidente mediante cursos que se imparten de manera informal por toda Europa y América. Como investigadores educativos resultaría empobrecedor cerrar los ojos ante este fenómeno. Así pues, con estas pruebas empíricas esperamos abrir nuevos caminos de investigación que enriquezcan nuestras prácticas educativas.

\section{REFERENCIAS}

Al Huang. (2002). La esencia del Tai Chi. Málaga: Editorial Sirio.

Calle. (2001). El arte de meditar. Madrid: Edaf.

Chang. (1970) Creativity and Taoism. NewYork: Harper \& Row Publishers.

Cheng. (2002). Historia y pensamiento chino. Barcelona: Ediciones Bellaterra.

Cheng. (2001). Los trece capitulos del Tai-Chi Chuan del maestro Cheng (El arte de la armonía). Madrid: Ediciones Tao.

Gardner. (1994). Educación artística y desarrollo humano. Barcelona: Paidós.

Gardner. (1995). Inteligencias múltiples. La teoría en la práctica. Barcelona: Paidós.

Punset. (2009). Meditación y aprendizaje. Redes, 50. Recuperado el 4 de marzo de 2010, de http://www.redesparalaciencia.com/1799/redes/2009/redes-50-meditación-yaprendizaje. 


\section{Silvia Viñao}

Doctora por la Universidad de Granada en Pedagogía (2011) y licenciada en Bellas Artes (Granada 1995). Ha sido profesora de la Universidad Católica de Murcia del 2001 al 2007. Ha realizado 27 exposiciones individuales a nivel nacional y más de 60 colectivas a nivel nacional e internacional, además de otras actividades en campos artísticos tales como la ilustración, el diseño, el video o diversas intervenciones artísticas. 